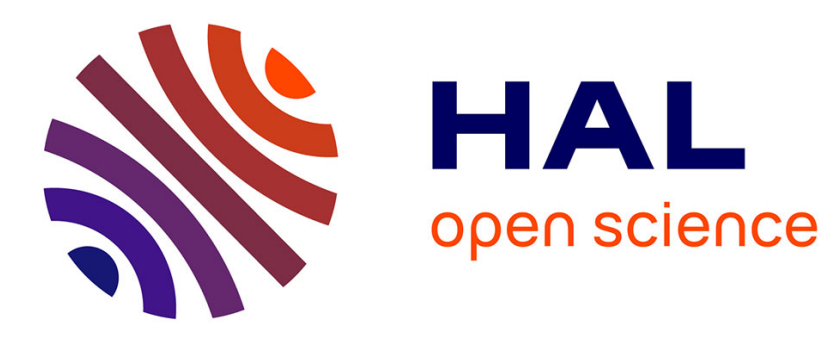

\title{
Matrices dermiques acellulaires et matrices synthétiques: évolution des pratiques en reconstruction mammaire immédiate par prothèse
}

\author{
R. Billon, B. Hersant, R. Bosc, J.P. Meningaud
}

\section{- To cite this version:}

R. Billon, B. Hersant, R. Bosc, J.P. Meningaud. Matrices dermiques acellulaires et matrices synthétiques: évolution des pratiques en reconstruction mammaire immédiate par prothèse. Gynécologie Obstétrique Fertilité \& Sénologie, 2019, 47, pp.311 - 316. 10.1016/j.gofs.2019.01.010 hal-03486611

\section{HAL Id: hal-03486611 \\ https://hal.science/hal-03486611}

Submitted on 20 Dec 2021

HAL is a multi-disciplinary open access archive for the deposit and dissemination of scientific research documents, whether they are published or not. The documents may come from teaching and research institutions in France or abroad, or from public or private research centers.
L'archive ouverte pluridisciplinaire HAL, est destinée au dépôt et à la diffusion de documents scientifiques de niveau recherche, publiés ou non, émanant des établissements d'enseignement et de recherche français ou étrangers, des laboratoires publics ou privés.

\section{(c) (1) $\$$}

Distributed under a Creative Commons Attribution - NonCommerciall 4.0 International 


\section{Mise au point}

\section{Acellular dermal matrix and synthetic mesh in implant-based immediate breast reconstruction : current concepts}

\section{Matrices dermiques acellulaires et matrices synthétiques : évolution des pratiques en reconstruction mammaire immédiate par prothèse}

Billon $\mathrm{R}^{1,2}$, Hersant $\mathrm{B}^{1,2}$, Bosc $\mathrm{R}^{1,2}$, Meningaud $\mathrm{JP}^{1}$

Raphaëlle Billon, M.D.

Barbara Hersant, M.D.

Romain Bosc, M.D.

Jean-Paul Meningaud, M.D., Ph.D.

${ }^{1}$ Department of Plastic, Reconstructive, Aesthetic and Maxillo-Facial Surgery, Henri Mondor University Hospital; UPEC, University Paris-Est Créteil, 51, avenue du Maréchal-de-Lattrede-Tassigny, 94010 Créteil, France

${ }^{2}$ Centre Sein Henri Mondor, Henri Mondor University Hospital, 51, avenue du Maréchal-deLattre-de-Tassigny, 94010 Créteil, France

Corresponding author :

Dr Raphaëlle Billon

Department of Plastic, Reconstructive, Aesthetic and Maxillo-Facial Surgery

Henri Mondor University Hospital

51, avenue du Maréchal de Lattre de Tassigny

94000, Créteil, France

Tel: 0149812533 Fax: 0149812532

billonraphaelle@gmail.com 


\section{- Introduction}

Les pratiques en reconstruction mammaire sont en constante évolution. Depuis l'introduction de la mastectomie avec conservation de l'étui cutané et sacrifice de la plaque aréolo-mamelonnaire (PAM) ou « skin-sparing mastectomy» (SSM) en 1991 par Toth et Lappert [1], la reconstruction mammaire immédiate (RMI) s'est largement développée. Les résultats oncologiques sont équivalents à ceux de la mastectomie radicale modifiée $[2,3]$. Elle respecte un maximum de peau et conserve l'intégrité du sillon sous-mammaire permettant une reconstruction optimale. Un lambeau d'avancement abdominal peut y être associé, corrigeant le déficit cutané créé par le sacrifice de la PAM en recrutant la peau de l'abdomen. La reconstruction du sillon sous-mammaire doit alors être précise afin d'éviter un néo-sillon mal positionné ou mal défini. Plusieurs techniques ont été décrites dont la technique du « hamac» qui permet une définition régulière du néo-sillon [4-6].

La mastectomie conservatrice de la PAM ou « nipple sparing mastectomy »(NSM), développée depuis les années 2000, est de plus en plus utilisée en raison de ses avantages esthétiques [7, 8]. Cette technique conserve l'intégralité de l'étui cutané. Il n'est donc pas nécessaire d'apporter de la peau supplémentaire par l'intermédiaire d'une expansion cutanée ou d'un lambeau. Le sillon sous-mammaire est conservé, la projection du sein reconstruit est maintenue et il n'est pas nécessaire de reconstruire la PAM. Si elle est acceptée en chirurgie prophylactique, la sécurité oncologique en situation curative est encore débattue, les études sur le sujet étant de faible niveau de preuve scientifique avec une durée de suivi insuffisante $[9,10]$. Les risques d'envahissement occulte de la PAM et de récidive locale sont encore incertains. Actuellement, la conservation de la PAM en RMI thérapeutique ne peut être envisagée que dans le cadre d'un essai clinique, pour les carcinomes in situ étendus voir 
certains carcinomes infiltrants de petite taille avec une indication de mastectomie en l'absence de facteurs de mauvais pronostic et à condition que la lésion soit située à distance de la PAM.

Enfin, pour les seins volumineux et ptosés, une mastectomie avec réduction de l'étui cutané ou «skin reducing mastectomy » $(\mathrm{SRM})$ est réalisée selon un patron de Wise avec une cicatrice en $\mathrm{T}$ inversé. Le risque de complications est plus important conduisant souvent à la mise en place d'un expandeur pour éviter toute tension sur les lambeaux cutanés de mastectomie.

La RMI par implant reste la technique la plus utilisée en reconstruction mammaire dans le monde. La RMI peut être réalisée en un temps avec mise en place d'une prothèse définitive («direct-to-implant»), ou en deux temps («RMI différée », initialement décrite par Kronowitz en 2004) avec une phase d'expansion préalable par un expandeur [11].

La principale complication redoutée est la nécrose cutanée qui aboutirait à l'exposition de la prothèse et donc à l'échec de la reconstruction. Pour cette raison, les implants sont traditionnellement placés en position rétromusculaire pure ou rétromusculaire sous-fasciale. La loge est constituée par le muscle grand pectoral, dont les attaches inférieures sont laissées intactes, par le muscle serratus anterior ou son fascia pour la couverture latérale de l'implant et par la gaine des muscles grands droits ou fascia sous-pectoral pour sa couverture inférieure permettant ainsi une couverture musculaire totale $[12,13]$.

La compliance du fascia du muscle serratus anterior et du fascia sous-pectoral est cependant souvent insuffisante pour mettre en place la prothèse définitive d'emblée. Une expansion préalable de la loge rétromusculaire à l'aide d'un expandeur est alors nécessaire [12]. La couverture musculaire totale limite donc le volume des implants. Elle empêche 
l'expansion du pôle inférieur du sein. Elle diminue la projection du sein reconstruit. De plus, elle expose au risque de malposition de l'implant liée à la contrainte musculaire. Les résultats esthétiques sont donc altérés avec un aspect peu naturel du sein.

Le développement des matrices dermiques acellulaires (MDA) et des matrices synthétiques résorbables ou non résorbables a permis de contourner les limites classiques de la loge rétromusculaire sous-fasciale [14-19]. En 2005, Breuing et Warren [20] décrivent la première reconstruction mammaire prothétique utilisant une MDA d'origine cadavérique humaine $\left(\right.$ AlloDerm $\left.{ }^{\circledR}\right)$ suturée entre le bord inférieur du muscle grand pectoral et la paroi thoracique pour agrandir la loge prothétique. Depuis, un nombre croissant de produits sont disponibles, allant des MDA d'origine porcine ou bovine aux matrices synthétiques. Aux Etats-Unis, elles sont utilisées dans près de la moitié des reconstructions prothétiques [21]. L'objectif de cette mise au point est de présenter les avantages communs et spécifiques de ces matrices, de discuter du risque de complications et de leur rôle dans le regain d'intérêt pour la reconstruction prépectorale à travers une revue de la littérature.

\section{- Avantages des matrices}

Suturées entre le bord inférieur du muscle grand pectoral qui est désinséré et la paroi thoracique au niveau du sillon sous-mammaire, elles permettent d'agrandir à la demande la loge prothétique rétromusculaire. Celle-ci peut alors recevoir d'emblée la prothèse définitive idéale sans phase d'expansion préalable [15]. Ces dispositifs ont ainsi permis de réduire l'utilisation des expandeurs en RMI [22]. Des prothèses de volume plus important peuvent être placées sans qu'aucune tension ne soit exercée par l'implant sur le lambeau cutané de mastectomie ni sur la cicatrice (Figure 1). 
Grâce à un contrôle précis de la loge prothétique, les résultats esthétiques sont supérieurs en cas d'utilisation d'une matrice par rapport à la loge rétromusculaire classique chez les patientes ayant une RMI par prothèse $[18,23]$. La projection au pôle inférieur du sein est maintenue donnant un aspect plus naturel au sein [24, 25]. Elles permettent un contrôle précis et une bonne définition du sillon sous-mammaire et latéro-mammaire [18, 25]. Elles empêchent le déplacement de l'implant [23, 25]. Elles apportent un renfort tissulaire en recouvrant la partie inféro-latérale de la prothèse. Enfin, elles préviennent la rétraction secondaire du muscle grand pectoral vers le haut lorsque ses attaches inférieures sont sectionnées.

De nombreuses études suggèrent que l'utilisation des MDA pourrait réduire l'incidence des coques périprothétiques [26-29]. La prévention des capsulites rétractiles est un défi de la chirurgie reconstructrice mammaire depuis l'introduction des implants il y a plus d'un demisiècle. La physiopathologie des coques périprothétiques est encore mal connue. La capsule périprothétique est une réaction immunitaire physiologique de l'organisme à la présence d'un corps étranger. La coque est due à une amplification de ce phénomène avec épaississement et rétraction de la capsule. L'importance de cette réaction varie d'une patiente à l'autre, mais est majorée par l'inflammation locale comme lors d'une irradiation de la prothèse $[30,31]$ ou au cours d'un traitement par hormonothérapie [32]. Des résultats physiopathologiques récents suggèrent que l'utilisation des MDA pourrait servir de barrière à la réponse immunitaire et diminuer l'inflammation locale [26, 29]. En 2013, Cheng et al. ont décrit une nouvelle technique permettant de traiter les coques périprothétiques Baker III ou IV par une capsulectomie associée à la mise en place d'un nouvel implant qu'ils recouvraient 
complètement d'une MDA, avec d'excellents résultats [33]. Des résultats à plus long terme sont cependant nécessaires [27].

\section{- Les matrices dermiques acellulaires}

Les MDA sont conçues à partir de derme d'origine humaine (AlloDerm $\left.{ }^{\circledR}\right)$, porcine $\left(\right.$ Strattice $^{\circledR}$, Permacol $^{\mathrm{TM}}$ ) ou bovine (SurgiMend $\left.{ }^{\circledR}\right)($ Figure 2A). Les cellules antigéniques sont éliminées par différents moyens physiques (radiation) et chimiques, laissant en place une matrice extracellulaire riche en fibres de collagène. Elles peuvent ainsi être recolonisées par les cellules de l'hôte et revascularisées permettant leur intégration dans les tissus receveurs.

L'expérience de 331 RMI «direct-to-implant» effectuées avec AlloDerm ${ }^{\circledR}$ au Massachusetts General Hospital suggère que cette technique est associée à des résultats esthétiques favorables et à de faibles taux de complications chez des patients présentant des lambeaux cutanés épais et bien vascularisés après une SSM ou NSM [34]. De même, l'utilisation d'une matrice SurgiMend $^{\circledR}$ dans 147 RMI après SSM, NSM ou SRM, incluant 123 «direct-to-implant», réalisées entre 2013 et 2015 à Tel Aviv, Israël, a montré des résultats esthétiques supérieurs et des taux de complications similaires par rapport à la technique rétromusculaire traditionnelle après un suivi médian de 24 mois [35].

Les MDA peuvent être responsables d'un « red-breast syndrome » caractérisé par un érythème au pôle inférieur du sein, ou sur la totalité du sein en cas de position prépectorale, sans infection associée et régressant spontanément en quelques jours. Le « red-breast syndrome » pourrait résulter d'une réaction inflammatoire aux produits de conservation de la matrice ou être dû à la revascularisation de la matrice [36]. Le rinçage abondant de la matrice avant son implantation pourrait limiter sa survenue. Au moindre doute d'infection ou de sérome, une échographie avec ponction diagnostique et/ou évacuatrice doit être réalisée. 


\section{- Les matrices synthétiques résorbables}

Pour résoudre le problème lié au coût des MDA et à leur disponibilité, certains auteurs ont proposé d'utiliser des matrices synthétiques résorbables ou non résorbables.

Les mèches résorbables à l'acide polyglicolique ou Vycril ${ }^{\circledR}$ ont été utilisées comme alternative [14, 15, 37]. Elles ont l'avantage d'être largement disponibles, relativement peu coûteuses et résistantes à la formation de biofilm bactérien [38]. Tessler et al. [14] rapportent un taux de complications de $6,6 \%$ sur 76 reconstructions « direct-to-implant » avec seulement 1 cas de perte de l'implant (1,3\%). Les résultats esthétiques sont jugés excellents avec seulement 3 cas nécessitant une reprise chirurgicale pour augmentation de la taille de l'implant (3,9\%). Cependant, les mèches de Vicryl sont à résorption rapide (3 à 4 semaines) ce qui pourrait compromettre les résultats de la reconstruction sur le long terme. De plus, cette résorption rapide peut s'accompagner d'une réponse inflammatoire plus importante, favorisant la survenue de complications et de coques périprothétiques.

Une autre alternative aux MDA, à moindre coût, est le treillis synthétique résorbable à long terme (TIGR ${ }^{\circledR}$ Matrix) (Figure 2B) [39, 40]. À base de co-polymère de lactide, glycolide et triméthylène carbonate, il est constitué de deux types de fibres qui ont des durées de résorption différentes. Il est résistant pendant au moins 6 mois et totalement résorbé en 3 ans, permettant un renforcement tissulaire pendant la phase initiale de la cicatrisation et pendant le remodelage avec un dépôt de collagène plus important par rapport à un groupe contrôle avec treillis non résorbable type polypropylène [38, 41]. Pompei et al. [40] rapportent leur

expérience de l'utilisation de 60 matrices TIGR $^{\circledR}$ avec un taux de complications de 5,4\% (trois cas de nécrose cutanée avec un cas d'explantation, deux cas de séromes et un cas d'infection) et d'excellents résultats esthétiques. 
Ces matrices synthétiques présentent les mêmes avantages et semblent avoir des résultats esthétiques et des taux de complications similaires, avec une réduction importante du coût en comparaison aux MDA [39, 42-44].

\section{- Les matrices synthétiques non résorbables}

Bien que moins répandues, des matrices synthétiques non résorbables ont aussi été utilisées dans cette indication. Il y a peu de données cliniques sur leur utilisation dans la littérature. Des petites séries de cas utilisant des treillis à base de polypropylène $\left(\mathrm{PTFE}^{\circledR}\right)$ [45] ou polytetrafluoroethylene polyesther $\left(\right.$ Mersilene $\left.^{\circledR}\right)$ [46] ont été rapportées. La plus utilisée des matrices synthétiques non résorbables est composée d'une structure monofilament de

polypropylène revêtu de titane $\left(\right.$ TiLOOP $^{\circledR}$ Bra), dont l'utilisation dans la reconstruction mammaire a été approuvée en Europe en 2008 [47, 48]. Elle est disponible en différentes tailles en forme de soutien-gorge. Des études animales et humaines ont démontré une biocompatibilité améliorée par rapport aux treillis sans revêtement de titane, avec des preuves histologiques d'incorporation tissulaire [49, 50]. Dans une étude rétrospective multicentrique de 231 cas de reconstruction utilisant cette matrice non résorbable, le taux de complications était de $29 \%$ avec un taux de reprise chirurgicale de $13,4 \%$. Les taux de retrait de la matrice et de perte de l'implant étaient de 7,8\% et 8,7\% respectivement [47]. Une étude plus récente de 70 RMI utilisant une autre matrice non résorbable à base de polyesther (Surgimesh-PET ${ }^{\circledR}$ ) a révélé un taux d'infection de $10 \%$ [51].

Un effet indésirable rapporté est la survenue (estimée à $4 \%$ pour TiLOOP ${ }^{\circledR}$ Bra) de nodules granulomateux due à la réaction inflammatoire prolongée qui peut interférer avec la surveillance de récidive du cancer du sein [52]. Un autre inconvénient est leur rigidité qui peut créer des plis palpables et gênants à long terme chez les patientes avec des lambeaux 
cutanés fins [52]. Bien qu'il n'existe pas d'étude comparant les taux de complications entre matrices résorbables et non résorbables, le manque de données cliniques sur ces matrices non résorbables et le souhait pour la plupart des chirurgiens de réduire la quantité de matériel permanent implanté leur fait préféré les matrices résorbables ou biologiques.

\section{- Complications}

De nombreuses études évaluent le risque des complications liées à l'utilisation de ces matrices. La majorité de ces études sont des séries de cas sans groupe contrôle à faible niveau de preuve (IV).

Dans une revue de la littérature, Scheflan et Colwell [53] résument les taux de complications liées au MDA dans les études comparatives. Ils rapportent un taux d'infection allant de $3 \%$ à $28,9 \%$ et un taux de sérome allant de $1,5 \%$ à $29,9 \%$ selon les études.

Dans une méta-analyse incluant les études comparatives réalisées entre 2011 et 2014, Lee et Mun [54] ont comparé les résultats de l'utilisation d'une MDA avec la technique sousmusculaire traditionnelle. Un total de 23 études, dont un essai contrôlé randomisé et trois études de cohortes prospectives, représentant 6199 cas ont été analysées. L'utilisation d'une MDA a significativement accru les risques d'infection (RR 1,42, IC ${ }^{95 \%} 1,02-1,99$ ), de sérome (RR 1,41, IC ${ }^{95 \%} 1,12-1,78$ ), et de nécrose des lambeaux cutanés de mastectomie (RR 1,44, $\left.\mathrm{IC}^{95 \%} 1,11-1,87\right)$, mais n'a pas augmenté les risques de réintervention en urgence (RR 1,09, $\left.\mathrm{IC}^{95 \%} 0,63-1,90\right)$ ni de perte de l'implant (RR 1,00, IC $\left.{ }^{95 \%} 0,68-1,48\right)$. Les risques de capsulite rétractile ( $\left.\mathrm{RR} 0,26, \mathrm{IC}^{95 \%} 0,15-0,47\right)$ et de malposition de l'implant (RR 0,21, $\left.\mathrm{IC}^{95 \%} 0,07-0,59\right)$ ont été significativement réduits. L'utilisation d'une MDA permet la mise en place d'un implant de volume significativement plus important. Cependant la plupart de ces études incluent des RMI en un temps et en deux temps et n'ont pas analysé séparément les 
complications par sous-groupe en fonction de la procédure utilisée ni en fonction d'une radiothérapie post-opératoire.

Une autre méta-analyse incluant les études comparant l'utilisation des matrices synthétiques aux reconstructions sans matrices n'a pas mis en évidence de différence significative sur la survenue d'infection, de perte de l'implant ni d'autres complications [55].

\section{- Sélection des patientes}

La sélection des patientes est primordiale pour éviter les complications. Les facteurs de risque de complications bien identifiés sont un antécédent de radiothérapie, un tabagisme actif

et un $\mathrm{IMC} \geq 30[56,57]$. De même, un volume mammaire important $(>600 \mathrm{~g})$, une ptôse importante (grade III et IV) ou la réduction de l'étui cutané sont à risque de nécrose cutanée [58]. Le volume de l'implant doit être adapté pour éviter toute tension cutanée et la bonne vascularisation des lambeaux cutanés de mastectomie doit être vérifiée en peropératoire (clinique, vert d'indocyanine) [59]. Au moindre doute, un expandeur sera préféré.

\section{- Évolution vers la reconstruction prépectorale}

Le développement des matrices a joué un rôle décisif dans la résurgence des reconstructions prothétiques prépectorales. Elles peuvent en effet être utilisées en position prépectorale recouvrant alors totalement l'implant [60, 61]. Elles apportent un soutien supplémentaire qui maintient l'implant en place. La loge est parfaitement ajustée et l'implant ne peut pas tourner. L'avantage est d'éviter l'animation du sein lors des contractions du muscle grand pectoral et d'éviter les douleurs liées au décollement du muscle. 
Au total, 15 études ont été publiées sur les reconstructions mammaires prothétiques prépectorales avec implant recouvert par une matrice, soient 1196 procédures, avec un suivi allant de 1 à 136 mois [61-75]. Les résultats de ces études ont été colligés dans la métaanalyse de Tasoulis et al. [60]. On retrouve des résultats satisfaisants chez des patientes sélectionnées autant sur les taux de complications qui sont équivalents à la reconstruction rétropectorale que sur les résultats esthétiques qui sont équivalents voir supérieurs. Le taux de coque périprothétique serait également diminué par l'utilisation des MDA.

Trois études de cohortes comparatives [64, 73, 75] ont évalué les résultats de reconstructions prépectorales avec couverture complète de l'implant par une matrice en comparaison à ceux des reconstructions rétropectorales avec utilisation d'une matrice. Les auteurs ne rapportent pas de différence significative sur l'incidence des complications, notamment sur les taux d'explantation, de nécrose cutanée ou de la PAM, et de réinterventions. Par ailleurs, dans l'étude de Bernini et al. [73], aucune coque périprothétique Baker III/IV n'est survenue dans le groupe des reconstructions prépectorales tandis que leur incidence était de $12 \%$ dans le groupe des reconstructions rétropectorales $(\mathrm{P}<0,01)$ après un suivi médian de 25 mois. Le résultat esthétique était jugé supérieur dans le groupe des reconstructions prépectorales par 2 chirurgiens indépendants $(\mathrm{P}<0,01$, échelle de Likert à 5 niveaux). Les auteurs ne rapportent pas plus de plis, de visibilité ou de palpabilité de l'implant dans le groupe des reconstructions prépectorales. La satisfaction des patientes était par ailleurs évaluée à l'aide du questionnaire BREAST-Q. Les scores étaient significativement meilleurs dans le groupe des reconstructions prépectorales. Les autres études sont des séries de cas rétrospectives.

Le manque d'études comparatives prospectives, l'hétérogénéité des populations étudiées, les durées de suivi trop courtes et la diversité des matrices utilisées limitent encore la pertinence de ces résultats. Ces études montrent cependant que les risques classiquement 
associés aux reconstructions prothétiques prépectorales doivent être réévalués. La visibilité de l'implant peut être atténuée par l'utilisation d'une matrice ainsi que par des réinjections de graisse autologue.

Le risque d'exposition de l'implant est limité par une sélection active des patientes et par une technique rigoureuse de mastectomie. Pour Sigalove et al. [70] et Vidya et al. [67], la sélection des patientes doit être rigoureuse afin de limiter les complications et d'optimiser les résultats de la reconstruction prothétique prépectorale. Cette technique peut être proposée aux patientes présentant les critères suivants : non fumeuses ; IMC $<35 \mathrm{~kg} / \mathrm{m} 2$; pas d'irradiation prévue sur le sein; ptôse de grade I ou II selon la classification de Regnault et poids de résection anticipé < $500 \mathrm{~g}$; lambeau cutané de mastectomie bien vascularisé. Le choix de l'incision de mastectomie doit être adapté, la technique de dissection doit être rigoureuse avec clivage cutanéo-glandulaire et l'utilisation des écarteurs doit être restreinte et contrôlée. Au moindre doute, une évaluation peropératoire de la vitalité du lambeau cutané de mastectomie peut être effectuée à l'aide de l'angiographie au vert d'indocyanine [59].

\section{- Conclusions}

La revue de la littérature sur l'utilisation des matrices est difficile à interpréter en raison d'une grande hétérogénéité des études et de leur faible niveau de preuve scientifique.

En reconstruction mammaire immédiate, l'utilisation de matrices permet de placer en un temps l'implant définitif idéal. Elles permettent également d'améliorer la définition du pôle inférieur et du sillon sous-mammaire. Elles pourraient réduire le risque de coque périprothétique et ont contribué à la résurgence des reconstructions prépectorales. Cependant, 
l'absence de remboursement des matrices en reconstruction mammaire constitue encore un inconvénient majeur à l'expansion de cette technique.

La sélection appropriée des patientes et une technique chirurgicale rigoureuse sont indispensables afin d'éviter les complications liées à l'utilisation des matrices dermiques acellulaires ou synthétiques.

Des essais contrôlés qui analysent davantage l'impact de la radiothérapie, le type de matrice et le type de procédure (reconstruction rétropectorale et prépectorale) sont nécessaires.

\section{Déclaration d'intérêts}

Les auteurs déclarent ne pas avoir de liens d'intérêts. 


\section{Références bibliographiques}

1. Toth BA, Lappert P. Modified skin incisions for mastectomy: the need for plastic surgical input in preoperative planning. Plast Reconstr Surg. juin 1991;87(6):1048 $\square 53$.

2. Carlson GW, Bostwick J, Styblo TM, Moore B, Bried JT, Murray DR, et al. Skin-sparing mastectomy. Oncologic and reconstructive considerations. Ann Surg. mai 1997;225(5):570575; discussion 575-578.

3. Rivadeneira DE, Simmons RM, Fish SK, Gayle L, La Trenta GS, Swistel A, et al. Skinsparing mastectomy with immediate breast reconstruction: a critical analysis of local recurrence. Cancer J Sudbury Mass. oct 2000;6(5):331 $\square 5$.

4. Nava M, Quattrone P, Riggio E. Focus on the breast fascial system: a new approach for inframammary fold reconstruction. Plast Reconstr Surg. sept 1998;102(4):1034@45.

5. Bogetti P, Cravero L, Spagnoli G, Devalle L, Boriani F, Bocchiotti MA, et al. Aesthetic role of the surgically rebuilt inframammary fold for implant-based breast reconstruction after mastectomy. J Plast Reconstr Aesthetic Surg JPRAS. 2007;60(11):1225[32.

6. Sarfati B, Honart JF, Guimond C, Rimareix F. Inframammary fold reconstruction: the « hammock » technique. J Plast Reconstr Aesthetic Surg JPRAS. mars 2015;68(3):433?4. 
7. Simmons RM, Brennan M, Christos P, King V, Osborne M. Analysis of nipple/areolar involvement with mastectomy: can the areola be preserved? Ann Surg Oncol. mars 2002;9(2):165 $\square 8$.

8. Simmons RM, Hollenbeck ST, Latrenta GS. Areola-sparing mastectomy with immediate breast reconstruction. Ann Plast Surg. déc 2003;51(6):547 $\square 51$.

9. Mota BS, Riera R, Ricci MD, Barrett J, de Castria TB, Atallah ÁN, et al. Nipple- and areola-sparing mastectomy for the treatment of breast cancer. Cochrane Database Syst Rev. 29 2016;11:CD008932.

10. Lostumbo L, Carbine NE, Wallace J. Prophylactic mastectomy for the prevention of breast cancer. Cochrane Database Syst Rev. 10 nov 2010;(11):CD002748.

11. Kronowitz SJ, Hunt KK, Kuerer HM, Babiera G, McNeese MD, Buchholz TA, et al. Delayed-immediate breast reconstruction. Plast Reconstr Surg. mai 2004;113(6):1617 $\square 28$.

12. Saint-Cyr M, Dauwe P, Wong C, Thakar H, Nagarkar P, Rohrich RJ. Use of the serratus anterior fascia flap for expander coverage in breast reconstruction. Plast Reconstr Surg. avr 2010;125(4):1057 $\square 64$.

13. Saint-Cyr M, Nagarkar P, Wong C, Thakar H, Dauwe P, Rohrich RJ. The pedicled subpectoral fascia flap for expander coverage in postmastectomy breast reconstruction: a novel technique. Plast Reconstr Surg. mai 2010;125(5):1328 $\square 34$. 
14. Tessler O, Reish RG, Maman DY, Smith BL, Austen WG. Beyond biologics: absorbable mesh as a low-cost, low-complication sling for implant-based breast reconstruction. Plast Reconstr Surg. févr 2014;133(2):90e $\square$ 9e.

15. Meyer Ganz O, Tobalem M, Perneger T, Lam T, Modarressi A, Elias B, et al. Risks and benefits of using an absorbable mesh in one-stage immediate breast reconstruction: a comparative study. Plast Reconstr Surg. mars 2015;135(3):498e $\square 507$ e.

16. Faulkner HR, Neumann R, Tessler O, Maman D, Smith BL, Austen WG. Three Years Experience with Absorbable Mesh in Single-Stage Breast Reconstruction: A Cost-Effective Alternative. Plast Reconstr Surg. oct 2015;136(4 Suppl):112 $\square 3$.

17. Salzberg CA. Nonexpansive immediate breast reconstruction using human acellular tissue matrix graft (AlloDerm). Ann Plast Surg. juill 2006;57(1):1 $\square 5$.

18. Spear SL, Parikh PM, Reisin E, Menon NG. Acellular dermis-assisted breast reconstruction. Aesthetic Plast Surg. mai 2008;32(3):418 $\square 25$.

19. Sbitany H, Sandeen SN, Amalfi AN, Davenport MS, Langstein HN. Acellular dermisassisted prosthetic breast reconstruction versus complete submuscular coverage: a head-tohead comparison of outcomes. Plast Reconstr Surg. déc 2009;124(6):1735 $\square 40$.

20. Breuing KH, Warren SM. Immediate bilateral breast reconstruction with implants and inferolateral AlloDerm slings. Ann Plast Surg. sept 2005;55(3):232 $\square 9$. 
21. Gurunluoglu R, Gurunluoglu A, Williams SA, Tebockhorst S. Current trends in breast reconstruction: survey of American Society of Plastic Surgeons 2010. Ann Plast Surg. janv 2013;70(1):103 $\square 10$.

22. Choi M, Frey JD, Alperovich M, Levine JP, Karp NS. « Breast in a Day »: Examining Single-Stage Immediate, Permanent Implant Reconstruction in Nipple-Sparing Mastectomy. Plast Reconstr Surg. août 2016;138(2):184e $\square 91$ e.

23. Vardanian AJ, Clayton JL, Roostaeian J, Shirvanian V, Da Lio A, Lipa JE, et al. Comparison of implant-based immediate breast reconstruction with and without acellular dermal matrix. Plast Reconstr Surg. nov 2011;128(5):403e $\square 410$ e.

24. Breuing $\mathrm{KH}$, Warren SM. Immediate bilateral breast reconstruction with implants and inferolateral AlloDerm slings. Ann Plast Surg. sept 2005;55(3):232 $\square 9$.

25. Breuing $\mathrm{KH}$, Colwell AS. Inferolateral AlloDerm hammock for implant coverage in breast reconstruction. Ann Plast Surg. sept 2007;59(3):250 $\square 5$.

26. Basu CB, Leong M, Hicks MJ. Acellular cadaveric dermis decreases the inflammatory response in capsule formation in reconstructive breast surgery. Plast Reconstr Surg. déc 2010;126(6):1842 $\square 7$.

27. Salzberg CA, Ashikari AY, Berry C, Hunsicker LM. Acellular Dermal Matrix-Assisted Direct-to-Implant Breast Reconstruction and Capsular Contracture: A 13-Year Experience. Plast Reconstr Surg. août 2016;138(2):329 $\square 37$. 
28. Lardi AM, Ho-Asjoe M, Junge K, Farhadi J. Capsular contracture in implant based breast reconstruction-the effect of porcine acellular dermal matrix. Gland Surg. févr $2017 ; 6(1): 49 \square 56$.

29. Leong M, Basu CB, Hicks MJ. Further evidence that human acellular dermal matrix decreases inflammatory markers of capsule formation in implant-based breast reconstruction. Aesthet Surg J. janv 2015;35(1):40 $\square 7$.

30. Vandeweyer E, Deraemaecker R. Radiation therapy after immediate breast reconstruction with implants. Plast Reconstr Surg. juill 2000;106(1):56-58; discussion 59-60.

31. Ascherman JA, Hanasono MM, Newman MI, Hughes DB. Implant reconstruction in breast cancer patients treated with radiation therapy. Plast Reconstr Surg. févr 2006;117(2):359 $\square 65$.

32. Billon R, Bosc R, Belkacemi Y, Assaf E, SidAhmed-Mezi M, Hersant B, et al. Impact of adjuvant anti-estrogen therapies (tamoxifen and aromatase inhibitors) on perioperative outcomes of breast reconstruction. J Plast Reconstr Aesthetic Surg JPRAS. nov 2017;70(11):1495 $\square 504$.

33. Cheng A, Lakhiani C, Saint-Cyr M. Treatment of capsular contracture using complete implant coverage by acellular dermal matrix: a novel technique. Plast Reconstr Surg. sept 2013;132(3):519 $\square 29$. 
34. Colwell AS, Damjanovic B, Zahedi B, Medford-Davis L, Hertl C, Austen WG. Retrospective review of 331 consecutive immediate single-stage implant reconstructions with acellular dermal matrix: indications, complications, trends, and costs. Plast Reconstr Surg. déc 2011;128(6):1170 $\square 8$.

35. Scheflan M, Grinberg-Rashi H, Hod K. Bovine Acellular Dermal Matrix in Immediate Breast Reconstruction: A Retrospective, Observational Study with SurgiMend. Plast Reconstr Surg. 2018;141(1):1e $\square 10 \mathrm{e}$.

36. Israeli R. Complications of acellular dermal matrices in breast surgery. Plast Reconstr Surg. nov 2012;130(5 Suppl 2):159S $\square 72$ S.

37. Loustau HD, Mayer HF, Sarrabayrouse M. Immediate prosthetic breast reconstruction: the ensured subpectoral pocket (ESP). J Plast Reconstr Aesthetic Surg JPRAS. 2007;60(11):1233 $\square 8$.

38. Nyame TT, Lemon KP, Kolter R, Liao EC. High-throughput assay for bacterial adhesion on acellular dermal matrices and synthetic surgical materials. Plast Reconstr Surg. nov 2011;128(5):1061 $\square 8$.

39. Becker H, Lind JG. The use of synthetic mesh in reconstructive, revision, and cosmetic breast surgery. Aesthetic Plast Surg. oct 2013;37(5):914 $\square 21$. 
40. Pompei S, Evangelidou D, Arelli F, Ferrante G. The Use of TIGR Matrix in Breast Aesthetic and Reconstructive Surgery: Is a Resorbable Synthetic Mesh a Viable Alternative to Acellular Dermal Matrices? Clin Plast Surg. janv 2018;45(1):65 $\square 73$.

41. Hjort H, Mathisen T, Alves A, Clermont G, Boutrand JP. Three-year results from a preclinical implantation study of a long-term resorbable surgical mesh with time-dependent mechanical characteristics. Hernia J Hernias Abdom Wall Surg. avr 2012;16(2):191 77.

42. Tessler O, Reish RG, Maman DY, Smith BL, Austen WG. Beyond biologics: absorbable mesh as a low-cost, low-complication sling for implant-based breast reconstruction. Plast Reconstr Surg. févr 2014;133(2):90e $\square$ 9e.

43. Meyer Ganz O, Tobalem M, Perneger T, Lam T, Modarressi A, Elias B, et al. Risks and benefits of using an absorbable mesh in one-stage immediate breast reconstruction: a comparative study. Plast Reconstr Surg. mars 2015;135(3):498e $\square 507$ e.

44. Faulkner HR, Neumann R, Tessler O, Maman D, Smith BL, Austen WG. Three Years Experience with Absorbable Mesh in Single-Stage Breast Reconstruction: A Cost-Effective Alternative. Plast Reconstr Surg. oct 2015;136(4 Suppl):112 $\square 3$.

45. Amanti C, Regolo L, Moscaroli A, Lo Russo M, Macchione B, Coppola M, et al. Use of mesh to repair the submuscolar pocket in breast reconstruction: a new possible technique. Il G Chir. oct 2002;23(10):391国. 
46. Rietjens M, Garusi C, Lanfrey E, Petit JY. [Cutaneous suspension: immediate breast reconstruction with abdominal cutaneous advancement using a non-resorptive mesh. Preliminary results and report of 28 cases]. Ann Chir Plast Esthet. avr 1997;42(2):177]82.

47. Dieterich M, Paepke S, Zwiefel K, Dieterich H, Blohmer J, Faridi A, et al. Implant-based breast reconstruction using a titanium-coated polypropylene mesh (TiLOOP Bra): a multicenter study of 231 cases. Plast Reconstr Surg. juill 2013;132(1):8e-19e.

48. Dieterich M, Faridi A. Biological Matrices and Synthetic Meshes Used in Implant-based Breast Reconstruction - a Review of Products Available in Germany. Geburtshilfe Frauenheilkd. nov 2013;73(11):1100]6.

49. Dieterich M, Dieterich H, Timme S, Reimer T, Gerber B, Stubert J. Using a titaniumcoated polypropylene mesh (TiLOOP(®) Bra) for implant-based breast reconstruction: case report and histological analysis. Arch Gynecol Obstet. juill 2012;286(1):27326.

50. Scheidbach H, Tannapfel A, Schmidt U, Lippert H, Köckerling F. Influence of titanium coating on the biocompatibility of a heavyweight polypropylene mesh. An animal experimental model. Eur Surg Res Eur Chir Forsch Rech Chir Eur. oct 2004;36(5):31397.

51. Baldelli I, Cardoni G, Franchelli S, Fregatti P, Friedman D, Pesce M, et al. Implant-Based Breast Reconstruction Using a Polyester Mesh (Surgimesh-PET): A Retrospective SingleCenter Study. Plast Reconstr Surg. juin 2016;137(6):931e-9e. 
52. Riggio E, Chifu C, Martelli G, Ferraris C. Can titanium mesh influence local recurrence management after implant-based breast reconstruction? SpringerPlus. 2015;4:482.

53. Scheflan M, Colwell AS. Tissue Reinforcement in Implant-based Breast Reconstruction. Plast Reconstr Surg Glob Open. août 2014;2(8):e192.

54. Lee K-T, Mun G-H. Updated Evidence of Acellular Dermal Matrix Use for ImplantBased Breast Reconstruction: A Meta-analysis. Ann Surg Oncol. févr 2016;23(2):600 $\square 10$.

55. Hallberg H, Rafnsdottir S, Selvaggi G, Strandell A, Samuelsson O, Stadig I, et al. Benefits and risks with acellular dermal matrix (ADM) and mesh support in immediate breast reconstruction: a systematic review and meta-analysis. J Plast Surg Hand Surg. 10 janv $2018 ; 1 \square 18$.

56. Israeli R. Complications of acellular dermal matrices in breast surgery. Plast Reconstr Surg. nov 2012;130(5 Suppl 2):159S $\square 72$ S.

57. Davila AA, Seth AK, Wang E, Hanwright P, Bilimoria K, Fine N, et al. Human Acellular Dermis versus Submuscular Tissue Expander Breast Reconstruction: A Multivariate Analysis of Short-Term Complications. Arch Plast Surg. janv 2013;40(1):19 $\square 27$.

58. Lardi AM, Ho-Asjoe M, Mohanna P-N, Farhadi J. Immediate breast reconstruction with acellular dermal matrix: factors affecting outcome. J Plast Reconstr Aesthetic Surg JPRAS. août 2014;67(8):1098 $\square 105$. 
59. Struk S, Honart J-F, Qassemyar Q, Leymarie N, Sarfati B, Alkhashnam H, et al. [Use of indocyanine green angiography in oncological and reconstructive breast surgery]. Ann Chir Plast Esthet. févr 2018;63(1):54 $\square 61$.

60. Tasoulis M-K, Iqbal FM, Cawthorn S, MacNeill F, Vidya R. Subcutaneous implant breast reconstruction: Time to reconsider? Eur J Surg Oncol J Eur Soc Surg Oncol Br Assoc Surg Oncol. sept 2017;43(9):1636 $\square 46$.

61. Salibian AH, Harness JK, Mowlds DS. Staged Suprapectoral Expander/Implant Reconstruction without Acellular Dermal Matrix following Nipple-Sparing Mastectomy. Plast Reconstr Surg. janv 2017;139(1):30 $\square 9$.

62. Downs RK, Hedges K. An Alternative Technique for Immediate Direct-to-Implant Breast Reconstruction-A Case Series. Plast Reconstr Surg Glob Open. juill 2016;4(7):e821.

63. Kobraei EM, Cauley R, Gadd M, Austen WG, Liao EC. Avoiding Breast Animation Deformity with Pectoralis-Sparing Subcutaneous Direct-to-Implant Breast Reconstruction. Plast Reconstr Surg Glob Open. mai 2016;4(5):e708.

64. Zhu L, Mohan AT, Abdelsattar JM, Wang Z, Vijayasekaran A, Hwang SM, et al. Comparison of subcutaneous versus submuscular expander placement in the first stage of immediate breast reconstruction. J Plast Reconstr Aesthetic Surg JPRAS. avr 2016;69(4):e7786. 
65. Schnarrs RH, Carman CM, Tobin C, Chase SA, Rossmeier KA. Complication Rates With Human Acellular Dermal Matrices: Retrospective Review of 211 Consecutive Breast Reconstructions. Plast Reconstr Surg Glob Open. nov 2016;4(11):e1118.

66. Berna G, Cawthorn SJ, Papaccio G, Balestrieri N. Evaluation of a novel breast reconstruction technique using the Braxon®acellular dermal matrix: a new muscle-sparing breast reconstruction. ANZ J Surg. juin 2017;87(6):493 $\square 8$.

67. Vidya R, Masià J, Cawthorn S, Berna G, Bozza F, Gardetto A, et al. Evaluation of the effectiveness of the prepectoral breast reconstruction with Braxon dermal matrix: First multicenter European report on 100 cases. Breast J. nov 2017;23(6):670 $\square 6$.

68. Becker H, Lind JG, Hopkins EG. Immediate Implant-based Prepectoral Breast Reconstruction Using a Vertical Incision. Plast Reconstr Surg Glob Open. juin 2015;3(6):e412.

69. Reitsamer R, Peintinger F. Prepectoral implant placement and complete coverage with porcine acellular dermal matrix: a new technique for direct-to-implant breast reconstruction after nipple-sparing mastectomy. J Plast Reconstr Aesthetic Surg JPRAS. févr 2015;68(2):162 $\square 7$.

70. Sigalove S, Maxwell GP, Sigalove NM, Storm-Dickerson TL, Pope N, Rice J, et al. Prepectoral Implant-Based Breast Reconstruction: Rationale, Indications, and Preliminary Results. Plast Reconstr Surg. févr 2017;139(2):287ロ $\square 94$. 
71. Woo A, Harless C, Jacobson SR. Revisiting an Old Place: Single-Surgeon Experience on Post-Mastectomy Subcutaneous Implant-Based Breast Reconstruction. Breast J. sept 2017;23(5):545 $\square 53$.

72. Caputo GG, Marchetti A, Dalla Pozza E, Vigato E, Domenici L, Cigna E, et al. SkinReduction Breast Reconstructions with Prepectoral Implant. Plast Reconstr Surg. juin 2016;137(6):1702 $\square 5$.

73. Bernini M, Calabrese C, Cecconi L, Santi C, Gjondedaj U, Roselli J, et al. Subcutaneous Direct-to-Implant Breast Reconstruction: Surgical, Functional, and Aesthetic Results after Long-Term Follow-Up. Plast Reconstr Surg Glob Open. déc 2015;3(12):e574.

74. Casella D, Calabrese C, Bianchi S, Meattini I, Bernini M. Subcutaneous Tissue Expander Placement with Synthetic Titanium-Coated Mesh in Breast Reconstruction: Long-term Results. Plast Reconstr Surg Glob Open. déc 2015;3(12):e577.

75. Casella D, Bernini M, Bencini L, Roselli J, Lacaria MT, Martellucci J, et al. TiLoop® Bra mesh used for immediate breast reconstruction: comparison of retropectoral and subcutaneous implant placement in a prospective single-institution series. Eur J Plast Surg. 2014;37(11):599 $\square 604$. 


\section{Légendes des figures}

Figure 1. Nipple Sparing Mastectomy gauche par voie sous-mammaire avec procédure du ganglion sentinelle pour un carcinome intracanalaire étendu et reconstruction mammaire immédiate par prothèse définitive et matrice synthétique TIGR $^{\circledR}$ Matrix (A) Suture de la matrice au bord inférieur du muscle grand pectoral qui a été désinséré (B) Mise en place de la prothèse et suture de la matrice au niveau du sillon sous-mammaire. Couverture de la partie inférieure et latérale de la prothèse par la matrice. $(\mathrm{C}, \mathrm{D})$ Photos pré-opératoires $(\mathrm{E}, \mathrm{F})$ Résultats à 2 mois post-opératoires : sillon sous-mammaire et latéro-mammaire bien définis et projection conservée du pôle inférieur du sein reconstruit.

Figure 2. (A) Matrice dermique acellulaire SurgiMend ${ }^{\circledR}$ fenêtrée après rinçage au sérum physiologique (B) Matrice synthétique résorbable à long terme TIGR $^{\circledR}$ Matrix. 
